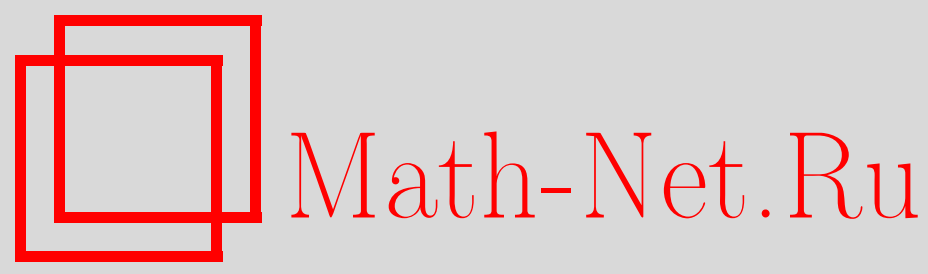

В. А. Садовничий, 3. Ю. Фазуллин, Асимптотика собственных чисел и формула следа возмущения оператора Лапласа на сфере $\mathbb{S}^{2}$, Матем. заметки, 2005, том 77, выпуск 3, 434448

DOI: https://doi.org/10.4213/mzm2504

Использование Общероссийского математического портала Math-Net.Ru подразумевает, что вы прочитали и согласны с пользовательским соглашением http://www.mathnet.ru/rus/agreement

Параметры загрузки:

IP : 54.80 .73 .141

26 апреля 2023 г., 14:58:07

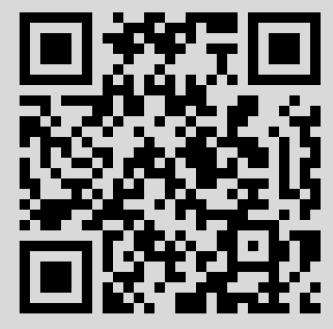




\title{
АСИМПТОТИКА СОБСТВЕННЫХ ЧИСЕЛ И ФОРМУЛА СЛЕДА ВОЗМУЩЕНИЯ ОПЕРАТОРА ЛАПЛАСА НА СФЕРЕ $\mathbb{S}^{2}$
}

\author{
В. А. Садовничий, З. Ю. Фазуллин
}

\begin{abstract}
В работе изучается асимптотика собственных чисел оператора Лапласа на сфеpe $\mathbb{S}^{2}$, возмущенного произвольным ограниченным оператором. Впервые для оператора в частных производных второго порядка получен главный член второй поправки теории возмущений. Установлена связь между коэффициентом второго члена асимптотики собственных чисел и формулой следов рассматриваемого оператора.

Библиография: 12 названий.
\end{abstract}

В работах [1], [2] Гийемин и Видом исследовали асимптотику собственных чисел оператора $L=-\Delta+V$ на сфере $\mathbb{S}^{n}$ с гладким нечетным потенциалом $V$ (т.е. $V(\tau s)=-V(s)$ для всякого $s \in \mathbb{S}^{n}$, где $\tau$ - антиподальное отображение) внутри одного кластера. В частности получена следующая оценка:

$$
\left|\mu_{k i}-\lambda_{k i}\right|=O\left(\frac{1}{k^{2}}\right), \quad i=1,2, \ldots, N_{k},
$$

где $N_{k}-$ кратность собственных чисел $\lambda_{k i}$ оператора $L_{0}=-\Delta, \mu_{k i}-$ собственные числа оператора $L$. В дальнейшем асимптотика спектра и регуляризованные следы для собственных чисел оператора $L$ на сфере $\mathbb{S}^{2}$ исследовались в работах [3], [4] в случае гладкого нечетного $V$ и в работах [5]-[8] в случае произвольного $V$. В частности, из результатов работы [7] следует, что при $V \in C^{2}\left(\mathbb{S}^{2}\right)$ справедлива асимптотическая формула

$$
\sum_{i=-k}^{k}\left[\mu_{k i}-\lambda_{k i}-c_{0}\right]=o\left(\frac{1}{k}\right),
$$

где $\lambda_{k i}=k(k+1)$ - собственные числа оператора $L_{0}$ кратности $(2 k+1)$ каждое. Следует заметить, что из доказательства теоремы 3 работы [7] вытекает более сильное утверждение, а именно, $o(1 / k)$ в формуле (1) можно заменить на $O\left(k^{-\alpha}\right), \alpha>1$, хотя это не отмечено. В работе [8] в случае, когда потенциал $V$ удовлетворяет условию Липшица по двум угловым переменным, показано, что правая часть формулы (1) есть $O\left(\ln k \cdot k^{-3 / 2}\right)$.

В настолщей статье мы предъявляем подробные доказательства более сильных утверждений, анонсированных нами в работе [9]. А именно, впервые для оператора в частных производных второго порядка получен второй член асимптотики собственных чисел. 
Итак, пусть $L_{0}=-\Delta$ - оператор Лапласа-Бельтрами на единичной сфере $\mathbb{S}^{2}$, действующий в гильбертовом пространстве $L^{2}\left(\mathbb{S}^{2}\right)$, а $V$ - оператор умножения на измеримую комплекснозначную функцию $V(\theta, \varphi)\left(\theta, \varphi-\right.$ сферические координаты) в $L^{2}\left(\mathbb{S}^{2}\right)$ и пусть $L=L_{0}+V$. Обозначим через $\lambda_{k}=k(k+1)$ и $\mu_{k i}, k=0,1, \ldots,-k \leqslant i \leqslant k$, собственные числа операторов $L_{0}$ и $L$ соответственно. Тогда справедлива следующая основная

ТЕОрема 1. Пусть $V(\omega)$ - произвольная, вообще говоря, комплексная функиия из класса $C^{3}\left(\mathbb{S}^{2}\right)$. Тогда для собственных чисел оператора L справедлива следующ, ая асимптотическая формула:

$$
\sum_{i=-k}^{k} \mu_{k i}=(2 k+1)\left[k(k+1)+c_{0}\right]+\frac{c_{1}}{k^{2}}+o\left(\frac{1}{k^{2}}\right)
$$

$2 \partial e$

$c_{0}=\frac{1}{4 \pi} \int_{\mathbb{S}^{2}} V(\omega) d \omega, \quad c_{1}=\frac{1}{32 \pi^{3}} \int_{\mathbb{S}^{2}} \int_{\mathbb{S}^{2}} \frac{V(\omega) V\left(\omega_{0}\right)}{\sqrt{1-\left(\vec{\omega}, \vec{\omega}_{0}\right)^{2}}} d \omega d \omega_{0}-\frac{1}{16 \pi} \int_{\mathbb{S}^{2}} V^{2}(\omega) d \omega$, $\left(\vec{\omega}, \vec{\omega}_{0}\right)$ - скалярное произведение векторов

$$
\vec{\omega}=(\cos \varphi \sin \theta, \sin \varphi \sin \theta, \cos \theta), \quad \vec{\omega}_{0}=\left(\cos \varphi_{0} \sin \theta_{0}, \sin \varphi_{0} \sin \theta_{0}, \cos \theta_{0}\right) .
$$

ДокАЗАТЕЛЬСтво. Пусть $R_{0}(z)=\left(L_{0}-z E\right)^{-1}, R(z)=(L-z E)^{-1}$ - резольвенты операторов $L_{0}$ и $L$ и $f_{k}^{(i)}=Y_{k}^{i}(\varphi, \theta)$ - сферические функции, ортонормированные собственные функции оператора $L_{0}$. Тогда в силу ограниченности оператора $V$ существует число $\rho$ такое, что $\|V\|<\rho \leqslant k+1$. Применяя оператор

$$
\operatorname{sp}\left(-\frac{1}{2 \pi i} \oint_{\left|\lambda_{k}-z\right|=\rho}(\cdot) z d z\right)
$$

( $\mathrm{sp}$ - след ядерного оператора) к обеим частям тождества

$$
\begin{aligned}
R(z)= & R_{0}(z)-R_{0}(z) V R_{0}(z)+\left(R_{0}(z) V\right)^{2} R_{0}(z) \\
& +\sum_{j=1}^{3}(-1)^{j}\left(R_{0}(z) V\right)^{j+2} R_{0}(z)+\left(R_{0}(z) V\right)^{6} R(z),
\end{aligned}
$$

используя теорему о вычетах получим

$$
\sum_{i=-k}^{k} \mu_{k i}=(2 k+1)\left[k(k+1)+c_{0}\right]+\alpha_{k}+\beta_{k}^{(1)}+\beta_{k}^{(2)}+\beta_{k}^{(3)}+O\left(\frac{1}{k^{3}}\right),
$$

где

$$
\begin{aligned}
\alpha_{k} & =\sum_{m=0, m \neq k}^{\infty} \frac{a_{m k}}{\lambda_{k}-\lambda_{m}}, \quad a_{m k}=\sum_{s=-k}^{k} \sum_{l=-m}^{m}\left|\left(V f_{m}^{(l)}, f_{k}^{(s)}\right)\right|^{2}, \\
\beta_{k}^{(j)} & =\frac{(-1)^{j+1}}{2 \pi i} \operatorname{sp} \oint_{\left|\lambda_{k}-z\right|=\rho}\left(R_{0}(z) V\right)^{j+2} R_{0}(z) z d z, \quad j=1,2,3 .
\end{aligned}
$$


Здесь мы еще воспользовались оценкой

$$
-\frac{1}{2 \pi i} \operatorname{sp} \oint_{\left|\lambda_{k}-z\right|=k+1}\left(R_{0}(z) V\right)^{6} R(z) z d z=O\left(\frac{1}{k^{3}}\right)
$$

в справедливости которой нетрудно убедиться, воспользовавшись следующими оценками для резольвент: $\left\|R_{0}(z)\right\| \leqslant(k+1)^{-1},\|R(z)\| \leqslant(k+1-\|V\|)^{-1}$ при $\left|\lambda_{k}-z\right|=k+1$.

Из равенства (3) заключаем: так как $\beta_{k}^{(j)}-$ последующие поправки теории возмущений и их порядок при $k \rightarrow \infty$ меньше порядка $\alpha_{k}$, для получения формулы $(2)$ достаточно доказать следующую теорему.

TEOPEMA 2. Пyсmь $k \gg 1 u V \in C^{3}\left(\mathbb{S}^{2}\right)$. Тогдa

$$
\alpha_{k}=\frac{c_{1}}{k^{2}}+o\left(\frac{1}{k^{2}}\right)
$$

Прежде чем приступить к доказательству теоремы 2 преобразуем числа $a_{k m}($ см. (4)). А именно, используя формулу сложения сферических гармоник, получаем

$$
\begin{aligned}
a_{k m}= & \frac{(2 k+1)(2 m+1)}{16 \pi^{2}} \\
& \times \int_{\mathbb{S}^{2}} \int_{\mathbb{S}^{2}} V(\varphi, \theta) V\left(\varphi_{0}, \theta_{0}\right) P_{k}(\cos \alpha) P_{m}(\cos \alpha) \sin \theta \sin \theta_{0} d \theta d \theta_{0} d \varphi d \varphi_{0},
\end{aligned}
$$

где $\cos \alpha=\left(\vec{\omega}, \vec{\omega}_{0}\right), P_{k}(\cos \alpha)$ - многочлены Лежандра, нормированные условием $P_{k}(1)=1$. Далее, введем унитарную матрицу

$$
T\left(\omega_{0}\right)=\left(\begin{array}{ccc}
\sin \varphi_{0} & \cos \theta_{0} \cos \varphi_{0} & \sin \theta_{0} \cos \varphi_{0} \\
-\cos \varphi_{0} & \cos \theta_{0} \sin \varphi_{0} & \sin \theta_{0} \sin \varphi_{0} \\
0 & -\sin \theta_{0} & \cos \theta_{0}
\end{array}\right)
$$

и вектор $\widetilde{\omega}=(\sin \alpha \cos \psi, \sin \alpha \sin \psi, \cos \alpha), 0 \leqslant \alpha \leqslant \pi, 0 \leqslant \psi \leqslant 2 \pi$. Тогда для чисел $a_{k m}$ получим следующую формулу:

$$
\begin{aligned}
& a_{k m}=\frac{(2 k+1)(2 m+1)}{16 \pi^{2}} \int_{0}^{\pi} q(\alpha) P_{k}(\cos \alpha) P_{m}(\cos \alpha) \sin \alpha d \alpha \\
& q(\alpha)=\int_{0}^{2 \pi} d \psi \int_{\mathbb{S}^{2}} V\left(\omega_{0}\right) V\left(T\left(\omega_{0}\right) \widetilde{\omega}\right) d \omega_{0} .
\end{aligned}
$$

Отметим, что $q^{\prime}(0)=q^{\prime}(\pi)=0$, в чем легко убедиться, используя формулы (5) и $(7)$, так как

$$
\int_{0}^{2 \pi} \sin \psi d \psi=\int_{0}^{2 \pi} \cos \psi d \psi=0
$$

Далее, так как

$$
\int_{0}^{\pi} P_{k}(\cos \alpha) P_{m}(\cos \alpha) \sin \alpha d \alpha=0
$$


при $m \neq k$, используя равенство [10, с. 41]

$$
\begin{aligned}
I(\alpha, m, k)= & \int_{0}^{\alpha} P_{k}(\cos \theta) P_{m}(\cos \theta) \sin \theta d \theta \\
= & \frac{1}{(m-k)(m+k+1)}\left[m P_{k}(\cos \alpha) P_{m-1}(\cos \alpha)\right. \\
& \left.\quad-k P_{m}(\cos \alpha) P_{k-1}(\cos \alpha)-(m-k) \cos \alpha P_{k}(\cos \alpha) P_{m}(\cos \alpha)\right]
\end{aligned}
$$

и интегрируя по частям в $(6)$, получим

$$
a_{k m}=\frac{(2 k+1)(2 m+1)}{16 \pi^{2}} \int_{0}^{\pi} q^{\prime}(\alpha) I(\alpha, m, k) d \alpha .
$$

Для изучения асимптотики $\alpha_{k}$ нам понадобятся следующие свойства многочленов Лежандра. Имеет место неравенство [11, с. 172]

$$
\sqrt{\sin \alpha}\left|P_{k}(\cos \alpha)\right|<\sqrt{\frac{2}{\pi k}}, \quad 0 \leqslant \alpha \leqslant \pi, \quad k=1,2, \ldots,
$$

и следующая асимптотическая формула [10, гл. VI, § 2], [12, с. 302]:

$$
\begin{aligned}
& P_{k}(\cos \alpha)= \frac{\cos [(k+1 / 2) \alpha-\pi / 4]}{\sin ^{1 / 2} \alpha}\left\{\frac{f_{0}}{k^{1 / 2}}+\frac{f_{1}}{k^{3 / 2}}+\frac{f_{2}}{k^{5 / 2}}+O\left(\frac{1}{k^{7 / 2}}\right)\right\} \\
&+\frac{\sin [(k+3 / 2) \alpha-\pi / 4]}{\sin ^{3 / 2} \alpha}\left\{\frac{g_{0}}{k^{3 / 2}}+\frac{g_{1}}{k^{5 / 2}}+O\left(\frac{1}{k^{7 / 2}}\right)\right\} \\
&+\frac{\cos [(k+5 / 2) \alpha-\pi / 4]}{\sin ^{5 / 2} \alpha}\left\{\frac{d_{0}}{k^{5 / 2}}+O\left(\frac{1}{k^{7 / 2}}\right)\right\}+\frac{O(1)}{k^{7 / 2} \sin ^{7 / 2} \alpha}, \\
& k \geqslant 1, \quad \varepsilon \leqslant \alpha \leqslant \pi-\varepsilon \quad \forall \varepsilon>0,
\end{aligned}
$$

где

$$
f_{0}=\sqrt{\frac{2}{\pi}}, \quad f_{1}=-\frac{3}{8} \sqrt{\frac{2}{\pi}}, \quad g_{0}=\frac{1}{8} \sqrt{\frac{2}{\pi}},
$$

$f_{2}, g_{1}, d_{0}$ - постоянные.

В дальнейшем нам нужна еще оценка суммы

$$
R_{k}=\sum_{m \neq k} \frac{k^{1-\beta} m^{1-\gamma}}{|m-k|^{q}(m+k)^{p}}
$$

при $k \rightarrow \infty$. С этой целью докажем лемму.

Лемма 1. Для $k \gg 1 u p+q+\gamma>2$ функиия $R_{k}$ имеет следуюшие оченки:

$$
\begin{array}{ll}
n p u \quad q=1 & R_{k}= \begin{cases}O\left(\frac{\ln k}{k^{p+\beta+\gamma-2}}\right), & \gamma \leqslant 2, \\
O\left(\frac{1}{k^{p+\beta}}\right), & \gamma>2,\end{cases} \\
n p u \quad q>1 & R_{k}= \begin{cases}O\left(\frac{1}{k^{p+\beta+\gamma-2}}\right), \\
O\left(\frac{1}{k^{p+\beta+\gamma-2}}\right)+O\left(\frac{1}{k^{p+\beta+q-1}}\right), & \gamma>2 .\end{cases}
\end{array}
$$


ДоказАТЕЛЬСтво. Разбивая $R_{k}$ на две суммы, имеем

$$
\begin{aligned}
R_{k} & =O\left(\left[\int_{1}^{k-1}+\int_{k+1}^{\infty}\right] \frac{k^{1-\beta} x^{1-\gamma} d x}{|x-k|^{q}(x+k)^{p}}\right) \\
& =\frac{1}{k^{q+p+\beta+\gamma-3}} O\left(\left[\int_{1 / k}^{1-1 / k}+\int_{1+1 / k}^{\infty}\right] \frac{\tau^{1-\gamma} d \tau}{|1-\tau|^{q}(1+\tau)^{p}}\right)
\end{aligned}
$$

в последнем равенстве мы воспользовались заменой переменных $x=k \tau$. Заметим, что при $q+p+\gamma>2$ второй интеграл сходится для $\tau \geqslant 2$. Следовательно, при $k \gg 1$ и $q=1$ имеем

$$
\left[\int_{1 / k}^{1-1 / k}+\int_{1+1 / k}^{\infty}\right] \frac{\tau^{1-\gamma} d \tau}{|1-\tau|^{q}(1+\tau)^{p}}=O(\ln k)+O\left(\left(\frac{1}{k}\right)^{2-\gamma}\right)
$$

а при $q>1$

$$
\left[\int_{1 / k}^{1-1 / k}+\int_{1+1 / k}^{\infty}\right] \frac{\tau^{1-\gamma} d \tau}{|1-\tau|^{q}(1+\tau)^{p}}=O\left(\left(\frac{1}{k}\right)^{2-\gamma}\right)+O\left(\left(\frac{1}{k}\right)^{1-q}\right)
$$

Из этих соотношений и равенства (12) легко следует утверждение леммы 1 . Лемма 1 доказана.

ДОКАЗАТЕЛЬСТВО ТЕОРЕМЫ 2. Используя формулы (4) и (9), числа $\alpha_{k}$ представим в следующем виде:

$$
\begin{aligned}
\alpha_{k}= & \sum_{m=0}^{N-1} \frac{a_{k m}}{\lambda_{k}-\lambda_{m}} \\
& -\frac{1}{16 \pi^{2}} \sum_{m=N, m \neq k}^{\infty} \frac{(2 k+1)(2 m+1)}{(m-k)(m+k+1)}\left(\int_{0}^{\varepsilon}+\int_{\pi-\varepsilon}^{\pi}\right) q^{\prime}(\alpha) I(\alpha, m, k) d \alpha \\
& -\frac{1}{16 \pi^{2}} \sum_{m=N, m \neq k}^{\infty} \frac{(2 k+1)(2 m+1)}{(m-k)(m+k+1)} \int_{\varepsilon}^{\pi-\varepsilon} q^{\prime}(\alpha) I(\alpha, m, k) d \alpha \\
= & \alpha_{k}^{(0)}+\alpha_{k}^{(1)}(\varepsilon)+\alpha_{k}^{(2)}(\varepsilon),
\end{aligned}
$$

где $N$ - достаточно большое фиксированное число.

ЗАмечАниЕ 1 . Разложение $\alpha_{k}$ на две суммы обусловлено тем, что в формуле (8) имеются многочлены Лежандра с индексом $m-1$, и при использовании их асимптотики из (11) имеем возможность применить формулу Тейлора для функции $(1-1 / m)^{\alpha}$ при $m \geqslant N$.

Итак, исследуем асимптотику каждого слагаемого в (13). Имеет место

ЛЕмма 2. При $k \gg 1$

$$
\alpha_{k}^{(1)}(\varepsilon)=O\left(\varepsilon^{3} \ln k\right)
$$


ДокАЗАТЕЛЬСтво. Пользуясь интегральным представлением функции $I(\alpha, k, m)$ из (8) и неравенством (10), получаем

$$
\left|\alpha_{k}^{(1)(\varepsilon)}\right| \leqslant \text { const } \sum_{m=N, m \neq k} \frac{k^{1 / 2} m^{1 / 2}}{|m-k|(m+k)}\left(\int_{0}^{\varepsilon}\left|q^{\prime}(\alpha)\right| \alpha d \alpha+\int_{\varepsilon}^{\pi-\varepsilon}\left|q^{\prime}(\alpha)\right|(\pi-\alpha) d \alpha\right) .
$$

Так как $q^{\prime}(0)=q^{\prime}(\pi)=0$, то интегралы в (14) имеют порядок $O\left(\varepsilon^{3}\right)$. А из леммы 1 следует, что

$$
\sum_{m=N, m \neq k} k^{1-1 / 2} m^{1-1 / 2}|m-k|^{-1}(m+k)^{-1}=O(\ln k) .
$$

Лемма 2 доказана.

Теперь исследуем асимптотику $\alpha_{k}^{(0)}(\varepsilon)$ из (13). Имеет место

Лемма 3. При $k \gg 1$

$$
\alpha_{k}^{(0)}(\varepsilon)=O\left(\varepsilon^{3 / 2} k^{-3 / 2}\right)+O\left(k^{-5 / 2}\right) .
$$

ДОКАЗАТЕЛЬСТВо. Для того чтобы воспользоваться представлением $a_{k m}$ из $(9)$ и (8) сначала изучим асимптотику первых двух слагаемых $\alpha_{k}^{(0)}(\varepsilon)$, т.е. при $m=0,1$, обозначив их через $\alpha_{k}^{(0)}(\varepsilon, m)$. Хорошо известно, что $P_{m}(\cos \alpha)=(\cos \alpha)^{m}, m=0,1$, поэтому имеем

$$
\begin{aligned}
\left|\alpha_{k}^{(0)}(\varepsilon, m)\right| \leqslant & \frac{\text { const }}{k}\left[\left|\left(\int_{0}^{\varepsilon}+\int_{\pi-\varepsilon}^{\pi}\right) q(\alpha)(\cos \alpha)^{m} P_{k}(\cos \alpha) \sin \alpha d \alpha\right|\right. \\
& \left.+\left|\int_{\varepsilon}^{\pi-\varepsilon} q(\alpha)(\cos \alpha)^{m} P_{k}(\cos \alpha) \sin \alpha d \alpha\right|\right]
\end{aligned}
$$

Теперь, используя неравенство (10) для первого слагаемого в (15) и асимптотику $P_{k}(\cos \alpha)$ из (11) с остатком $O\left(\sin ^{-3 / 2} \alpha \cdot k^{-3 / 2}\right)$ для второго слагаемого, интегрируя один раз по частям главный член асимптотики, получим, что

$$
\alpha_{k}^{(0)}(\varepsilon, m)=O\left(\varepsilon^{3 / 2} \cdot k^{-3 / 2}\right)+O\left(k^{-5 / 2}\right) .
$$

Далее, так как

$$
\int_{0}^{\pi}\left|\frac{q^{\prime}(\alpha)}{\sin \alpha}\right| d \alpha=O(1)
$$

воспользовавшись представлением для $a_{k m}$ из $(9)$ и (8), в силу неравенства (10) при $m \geqslant 2$ получим оценку

$$
\begin{aligned}
\left|\alpha_{k}^{(0)}(\varepsilon)\right| & \leqslant c_{1} \sum_{m=2}^{N-1} \frac{(2 k+1)(2 m+1)}{(m-k)^{2}(m+k+1)^{2}}\left[\frac{m^{1 / 2}}{k^{1 / 2}}+\frac{k^{1 / 2}}{(m-1)^{1 / 2}}+\frac{|m-k|}{k^{1 / 2}(m-1)^{1 / 2}}\right] \\
& =O\left(k^{-5 / 2}\right),
\end{aligned}
$$

откуда и из (16) следует доказательство леммы 3. 
Теперь приступим к изучению асимптотики $\alpha_{k}^{(2)}(\varepsilon)$. С этой целью подставим асимптотику для многочленов Лежандра из (11) в представление (8) для функции $I(\alpha, k, m)$. Проведя довольно громоздкие аналитические выкладки и при этом используя формулу Тейлора для функций $(1-1 / m)^{\gamma}$ и $(1-1 / k)^{\gamma}, m \geqslant N, k \geqslant 1$, из формулы (13) получим, что

$$
\begin{aligned}
& \alpha_{k}^{(2)}(\varepsilon)=-\frac{1}{32 \pi^{2}} \sum_{m=N, m \neq k} \frac{(2 k+1)(2 m+1)}{(m-k)^{2}(m+k+1)^{2}} \int_{\varepsilon}^{\pi-\varepsilon} q^{\prime}(\alpha) \\
& \times\left\{\sin (m-k) \alpha\left[\frac{a_{1}(m+k+1)}{k^{1 / 2} m^{1 / 2}}+\frac{a_{2}(m+k)^{2}}{k^{3 / 2} m^{3 / 2}}+\frac{a_{3}\left(m^{3}+k^{3}\right)}{k^{5 / 2} m^{5 / 2}}+O\left(\frac{m+k}{k^{\beta} m^{\gamma}}\right)\right]\right. \\
& +\frac{1}{\sin \alpha}\left\{\sin (m+k) \alpha\left[\frac{b_{1}^{(1)}(m-k)}{k^{3 / 2} m^{3 / 2}}+\frac{b_{3}^{(1)}\left(m^{3}-k^{3}\right)}{k^{5 / 2} m^{5 / 2}}+O\left(\frac{|m-k|}{k^{\beta} m^{\gamma}}\right)\right]\right. \\
& +\left[\sin ^{2} \alpha \sin (m+k) \alpha+\sin \alpha \cos (m+k) \alpha\right]\left[\frac{b_{1}^{(j)}(m-k)}{k^{1 / 2} m^{1 / 2}}\right. \\
& \left.+\frac{b_{2}^{(j)}\left(m^{2}-k^{2}\right)}{k^{3 / 2} m^{3 / 2}}+\frac{b_{4}^{(j)}(m-k)}{k^{3 / 2} m^{3 / 2}}+\frac{b_{3}^{(j)}\left(m^{3}-k^{3}\right)}{k^{5 / 2} m^{5 / 2}}+O\left(\frac{|m-k|}{k^{\beta} m^{\gamma}}\right)\right] \\
& +\cos \alpha \cos (m-k) \alpha\left[\frac{b_{1}^{(4)}(m-k)}{k^{3 / 2} m^{3 / 2}}\right. \\
& \left.\left.+\frac{b_{2}^{(4)}\left(m^{2}-k^{2}\right)}{k^{3 / 2} m^{3 / 2}}+\frac{b_{3}^{(3)}\left(m^{3}-k^{3}\right)}{k^{5 / 2} m^{5 / 2}}+O\left(\frac{|m-k|}{k^{\beta} m^{\gamma}}\right)\right]\right\} \\
& +\frac{1}{\sin ^{2} \alpha}\left\{\sin (m-k) \alpha\left[\frac{c_{1}(m+k)}{k^{3 / 2} m^{3 / 2}}+\frac{c_{3}\left(m^{3}+k^{3}\right)}{k^{5 / 2} m^{5 / 2}}+O\left(\frac{|m-k|}{k^{\beta} m^{\gamma}}\right)\right]\right. \\
& +[\cos (k+m+1) \alpha+\cos \alpha \cos (m+k+2) \alpha]\left[\frac{d_{1}^{(j)}(m-k)}{k^{3 / 2} m^{3 / 2}}\right. \\
& \left.\left.+\frac{d_{2}^{(j)}\left(m^{2}-k^{2}\right)}{k^{3 / 2} m^{3 / 2}}+\frac{d_{3}^{(j)}\left(m^{3}-k^{3}\right)}{k^{5 / 2} m^{5 / 2}}+O\left(\frac{|m-k|}{k^{\beta} m^{\gamma}}\right)\right]\right\} \\
& +\frac{1}{\sin ^{3} \alpha}\left\{[ \operatorname { s i n } ( m + k + 3 ) \alpha - \operatorname { c o s } ( m + k + 2 ) \alpha ] \left[\frac{l_{1}^{(j)}(m-k)}{k^{3 / 2} m^{3 / 2}}\right.\right. \\
& \left.\left.\left.+\frac{l_{3}^{(j)}\left(m^{3}-k^{3}\right)}{k^{5 / 2} m^{5 / 2}}+O\left(\frac{|m-k|}{k^{\beta} m^{\gamma}}\right)\right]\right\}+\frac{O(1)}{k^{\beta} m^{\gamma} \sin ^{4} \alpha}\right\} d \alpha,
\end{aligned}
$$

где $\beta=1 / 2,3 / 2,5 / 2,7 / 2, \beta \geqslant 1 / 2, \beta+\gamma=4, a_{i}, c_{i}, b_{i}^{(j)}, d_{i}^{(j)}, l_{i}^{(j)}$ - постоянные, верхний индекс $j$ которых указывает на то, что для каждой тригонометрической функции в квадратных скобках свои константы. Отметим, что

$$
a_{1}=f_{0}^{2}, \quad a_{2}=f_{0}\left[f_{1}+g_{0}\right] .
$$

ЛЕмма 4. При $k \gg 1 u q \in C^{3}[0, \pi]$ справедлива формула

$$
\alpha_{k}^{(2)}(\varepsilon)=\frac{1}{32 \pi^{3} k^{2}} \int_{0}^{\pi} q^{\prime}(\alpha)(\pi-\alpha) d \alpha+\frac{O\left(\varepsilon^{-2}\right)}{k^{7 / 2}}+\frac{O(\varepsilon)}{k^{3 / 2}}+\frac{O(\ln \varepsilon)}{k^{5 / 2}}+\frac{O(\varepsilon) \ln k}{k^{2}} .
$$


ДокАЗАТЕЛЬство. Исследуем асимптотическое поведение каждого слагаемого в (17). С этой целью заметим, что, так как $q^{\prime}(0)=q^{\prime}(\pi)=0$, то

$$
\int_{\varepsilon}^{\pi-\varepsilon}\left|q^{\prime}(\alpha)\right| \sin ^{-n} \alpha d \alpha= \begin{cases}O(1), & n \leqslant 1 \\ O(\ln \varepsilon), & n=2 \\ O\left(\varepsilon^{2-n}\right), & n \geqslant 3\end{cases}
$$

Более того, если $q \in C^{3}[0, \pi]$, то интегрированием по частям легко убеждаемся в справедливости оценок

$$
\int_{\varepsilon}^{\pi-\varepsilon} \frac{q^{\prime}(\alpha) \varphi(\alpha)}{\sin ^{n} \alpha}\left(\begin{array}{c}
\cos l \alpha \\
\sin l \alpha
\end{array}\right) d \alpha=\frac{O\left(\varepsilon^{1-n}\right)}{l}+\frac{O\left(\varepsilon^{-n}\right)}{l^{2}},
$$

где в качестве $\varphi(\alpha)$ может быть взята любая из функций: тождественная единица или $\cos \alpha$. Обозначим слагаемое $\alpha_{k}^{(2)}(\varepsilon)$, соответствующее в (17) члену $O(1) k^{-\beta} m^{-\gamma} \times$ $\sin ^{-4} \alpha$, через $\alpha_{k}^{(2,1)}(\varepsilon)$. Тогда, используя (19) и лемму 1 при $q=2, \gamma=1 / 2,3 / 2,5 / 2,7 / 2$, получим

$$
\alpha_{k}^{(2,1)}(\varepsilon)=\frac{O\left(\varepsilon^{-2}\right)}{k^{7 / 2}} .
$$

Далее, изучим слагаемые, соответствующие членам $O\left(|m \pm k| \cdot k^{-\beta} \cdot m^{-\gamma}\right)$ в (17), обозначив их через $\alpha_{k}^{(2,2)}(\varepsilon)$. Сначала проинтегрировав один раз по частям слагаемые, содержащие функции $\sin ^{3} \alpha$, затем используя оценку (19) и лемму 1 , получим, что

$$
\alpha_{k}^{(2,2)}(\varepsilon)=\frac{O(\ln \varepsilon)}{k^{5 / 2}}+\frac{O\left(\varepsilon^{-2}\right)}{k^{7 / 2}} .
$$

ЗАмЕЧАниЕ 2. В итоге мы положим $\varepsilon=k^{-\delta}, 2 / 3<\delta<3 / 4$. Следовательно, в оценке $\alpha_{k}^{(2,2)}(\varepsilon)$ и в дальнейшем главную часть асимптотики выбираем с учетом этого замечания.

Изучим слагаемые $\alpha_{k}^{2}(\varepsilon)$ с членами $(m-k) k^{-3 / 2} m^{-3 / 2}$ в (17), обозначив их через $\alpha_{k}^{(2,3)}(\varepsilon)$. Из леммы 1 при $q=1, p=2, \beta=\gamma=3 / 2$ и оценки (19) вытекает, что

$$
\alpha_{k}^{(2,3)}(\varepsilon)=\frac{O\left(\varepsilon^{-1}\right) \cdot \ln k}{k^{3}} .
$$

Теперь покажем, что

$$
\begin{aligned}
\alpha_{k}^{(2,4)}(\varepsilon)= & -\frac{b_{1}^{(j)}}{32 \pi^{2}} \sum_{m=N, m \neq k} \frac{(2 k+1)(2 m+1)}{(m-k)(m+k+1) k^{1 / 2} m^{1 / 2}} \\
& \times \int_{\varepsilon}^{\pi-\varepsilon} q^{\prime}(\alpha)[\sin \alpha \sin (m-k) \alpha+\cos (m+k) \alpha] d \alpha \\
= & \frac{O(\varepsilon \cdot \ln k)}{k^{2}}
\end{aligned}
$$

Действительно, два раза интегрируя по частям интеграл в (24), получим оценку (20) при $n=-1,0$. Тогда из леммы 1 при $q=1, \beta=\gamma=1 / 2, p=3,4$, учитьвая замечание 2 , получим оценку (24). Исследуем слагаемые $\alpha_{k}^{2}(\varepsilon)$, содержащие члены с 
$\left(m^{2}-k^{2}\right) m^{-3 / 2} k^{-3 / 2}$ в (17). Обозначим через $\alpha_{k}^{(2,5)}(\varepsilon)$ те из этих слагаемых, которые содержат $\sin (m+k+l) \alpha$ и $\cos (m+k+l) \alpha, l=0,1,2$. Тогда, один раз интегрируя по частям и воспользовавшись леммой 1 при $p=2, q=1, \beta=\gamma=3 / 2$, получим следующую оценку:

$$
\alpha_{k}^{(2,5)}(\varepsilon)=\frac{O\left(\varepsilon^{-1} \ln k\right)}{k^{3}} .
$$

Для изучения асимптотики слагаемого

$$
\begin{aligned}
\alpha_{k}^{(2,6)}(\varepsilon)= & -\frac{b_{2}^{(4)}}{32 \pi^{2}} \sum_{m=N, m \neq k} \frac{(2 k+1)(2 m+1)}{(m-k)^{2}(m+k+1)^{2}} \frac{\left(m^{2}-k^{2}\right)}{k^{1 / 2} m^{1 / 2}} \\
& \times \int_{\varepsilon}^{\pi-\varepsilon} q^{\prime}(\alpha) \frac{\cos \alpha \cos (m-k) \alpha}{\sin \alpha} d \alpha
\end{aligned}
$$

(так как $m+k \gg 1$ ) заметим, что

$$
\begin{aligned}
& \frac{(2 k+1)(2 m+1)}{(m-k)^{2}(m+k+1)^{2}} \frac{\left(m^{2}-k^{2}\right)}{k^{1 / 2} m^{1 / 2}} \\
& \quad=\frac{4 k m}{(m-k)(m+k) k^{3 / 2} m^{3 / 2}}+O\left(\frac{k^{-3 / 2} m^{-3 / 2}}{|m-k|}\right)+O\left(\frac{k^{-1 / 2} m^{-1 / 2}}{|m-k|(m+k)^{2}}\right) .
\end{aligned}
$$

Далее обозначим через $\widehat{\alpha}_{k}^{(2,6)}(\varepsilon)$ часть $\alpha_{k}^{(2,6)}(\varepsilon)$, соответствующую первому слагаемому в правой части $(26)$, а через $\widehat{\widehat{\alpha}}_{k}^{(2,6)}(\varepsilon)$ - второму и третьему. Тогда из леммы 1 и оценки (20) следует, что

$$
\widehat{\widehat{\alpha}}_{k}^{(2,6)}(\varepsilon)=O\left(k^{-5 / 2}\right) .
$$

Для изучения $\widehat{\alpha}_{k}^{(2,6)}(\varepsilon)$ положим $m-k=l_{\text {и }}$

$$
\widehat{b}_{l}=\int_{\varepsilon}^{\pi-\varepsilon} \frac{q^{\prime}(\alpha) \cos \alpha \cos l \alpha}{\sin \alpha} d \alpha
$$

и представим его в следующем виде:

$$
\begin{aligned}
\widehat{\alpha}_{k}^{(2,6)}(\varepsilon)= & -\frac{b_{2}^{(4)}}{8 \pi^{2}} \sum_{l=1}^{\left[k^{\sigma}\right]} \frac{\widehat{b}_{l}(\varepsilon)}{k^{1 / 2} l}\left[\frac{(k+l)^{-1 / 2}}{(2 k+l)}-\frac{(k-l)^{-1 / 2}}{(2 k-l)}\right] \\
& +\frac{b_{2}^{(4)}}{8 \pi^{2}} \sum_{l=\left[k^{\sigma}\right]+1}^{k-N} \frac{\widehat{b}_{l}(\varepsilon)}{k^{1 / 2} l} \frac{(k-l)^{-1 / 2}}{l(2 k-l)}-\frac{b_{2}^{(4)}}{8 \pi^{2}} \sum_{l=\left[k^{\sigma}\right]+1}^{\infty} \frac{\widehat{b}_{l}(\varepsilon)}{k^{1 / 2} l} \frac{(k+l)^{-1 / 2}}{l(2 k+l)} \\
= & \gamma_{1}(k, \varepsilon)+\gamma_{2}(k, \varepsilon)+\gamma_{3}(k, \varepsilon),
\end{aligned}
$$

где $0<\sigma<1$, значение которого выбираем в дальнейшем.

Исследуем $\gamma_{1}(k, \varepsilon)$. Применяя формулу Тейлора для функции

$$
f(x)=\frac{(1+2 x)^{-1 / 2}}{1+x}-\frac{(1-2 x)^{-1 / 2}}{1-x}
$$


при $x=l /(2 k)$ и оценку $(20)$ при $n=1$, получаем, что

$$
\gamma_{1}(k, \varepsilon)=O\left(\varepsilon^{-1} k^{-3}\right) .
$$

В силу (20) нетрудно убедиться, что

$$
\gamma_{2}(k, \varepsilon)=\frac{O\left(\varepsilon^{-1}\right)}{k^{2+2 \sigma}}+\frac{O(1)}{k^{2+\sigma}}, \quad \gamma_{3}(k, \varepsilon)=\frac{O\left(\varepsilon^{-1}\right)}{k^{2+2 \sigma}}+\frac{O(1)}{k^{2+\sigma}} .
$$

Таким образом, из (27)-(30) заключаем, что

$$
\alpha_{k}^{(2,6)}(\varepsilon)=O\left(k^{-5 / 2}\right)+O\left(k^{-(\sigma+2)}\right)+O\left(\varepsilon^{-1} k^{-(2+2 \sigma)}\right)+O\left(\varepsilon^{-1} k^{-3}\right) .
$$

Теперь изучим слагаемые в $\alpha_{k}^{2}(\varepsilon)$, содержашие члены $\left(m^{3}-k^{3}\right) k^{-5 / 2} m^{-5 / 2}$ в $(17)$, обозначив их через $\alpha_{k}^{(2,7)}(\varepsilon)$. Один раз интегрируя по частям интегралы в слагаемых с множителями $\sin (m+k+l) \alpha$ и $\cos (m+k+l) \alpha, l=0,1,2$, затем используя оценку (19) и лемму 1 , убеждаемся, что они имеют порядок $O\left(\varepsilon^{-2} k^{-7 / 2}\right)$. А для слагаемого $\left(m^{3}-k^{3}\right) k^{-5 / 2} m^{-5 / 2} \sin ^{-1} \alpha \cos \alpha \cos (m-k) \alpha$ в (17) из оценки (20) и леммы 1 получим оценку порядка $O\left(k^{-5 / 2}\right)$. Таким образом,

$$
\alpha_{k}^{(2,7)}(\varepsilon)=O\left(\varepsilon^{-2} k^{-7 / 2}\right)+O\left(k^{-5 / 2}\right) .
$$

Итак, остается оценить слагаемые $\alpha_{k}^{2}(\varepsilon)$ при $\sin (m-k) \alpha$ и $\sin (m-k) \alpha \sin ^{-2} \alpha$ в (17). Обозначив их соответственно $\alpha_{k}^{(2,8)}(\varepsilon)$ и $\alpha_{k}^{(2,9)}(\varepsilon)$, после несложных преобразований получим

$$
\begin{aligned}
\alpha_{k}^{(2,8)}(\varepsilon)=- & \frac{1}{32 \pi^{2}} \sum_{m=N, m \neq k}^{\infty} \int_{\varepsilon}^{\pi-\varepsilon} \frac{q^{\prime}(\alpha) \sin (m-k) \alpha}{(m-k)^{2}} d \alpha \\
& \times\left\{\frac{\tau_{0}}{k^{1 / 2} m^{1 / 2}}+\frac{\tau_{1} k^{1 / 2} m^{1 / 2}}{m+k}+\frac{\tau_{2}(m+k)}{k^{3 / 2} m^{3 / 2}}\right. \\
& \left.+\frac{\tau_{3}}{(m+k) k^{1 / 2} m^{1 / 2}}+\frac{\tau_{4}\left(m^{3}+k^{3}\right)}{k^{3 / 2} m^{3 / 2}(m+k)^{2}}\right\}+O\left(\frac{1}{k^{5 / 2}}\right) \\
= & \sum_{i=0}^{4} A_{i}(k, \varepsilon)+O\left(\frac{1}{k^{5 / 2}}\right), \\
\alpha_{k}^{(2,9)}(\varepsilon)= & \frac{1}{32 \pi^{2}} \sum_{m=N, m \neq k}^{\infty} \int_{\varepsilon}^{\pi-\varepsilon} \frac{q^{\prime}(\alpha) \sin (m-k) \alpha}{\sin ^{2} \alpha(m-k)^{2}} d \alpha \\
& \times\left\{\frac{\xi_{1}\left(m^{3}+k^{3}\right)}{(m+k) k^{1 / 2} m^{1 / 2}}+\frac{\xi_{2}}{k^{3 / 2} m^{3 / 2}(m+k)^{2}}\right\}+O\left(\frac{\ln \varepsilon}{k^{5 / 2}}\right) \\
= & \sum_{i=1}^{2} B_{i}(k, \varepsilon)+O\left(\frac{\ln \varepsilon}{k^{5 / 2}}\right),
\end{aligned}
$$

где $\tau_{i}, \xi_{i}$ - постоянные, причем (см. (18) и (11))

$$
\tau_{0}=2 a_{1}+4 a_{2}=\frac{1}{\pi}
$$


Отметим, что в $O\left(k^{-5 / 2}\right)$ и $O\left(k^{-5 / 2} \ln \varepsilon\right)$ включены слагаемые из $(17)$, которые после преобразования оценены с помощью леммы 1 и оценок (19). Асимптотика каждой суммы в $(33)$ и $(34)\left(A_{i}(k, \varepsilon)\right.$ и $\left.B_{i}(k, \varepsilon)\right)$ изучаются по следующей схеме. Полагаем $m-k=l$; сумма разбивается на три слагаемых: в первом слагаемом индекс суммирования $l$ меняется от $-\left[k^{\sigma}\right]$ до $\left[k^{\sigma}\right]$ и $l \neq 0$, во втором $-N-k \leqslant l \leqslant-\left[k^{\sigma}\right]-1$, в третьем $\left[k^{\sigma}\right]+1 \leqslant l \leqslant \infty, 0<\sigma<1$, и исследуется асимптотика каждого слагаемого. Отметим, что число $\sigma$ выбирается из условия, чтобы при $k \rightarrow \infty$ иметь порядок убывания $o\left(k^{-2}\right)$.

Итак, для первого слагаемого $A_{0}(k, \varepsilon)$ в $(33)$ с учетом значения $\tau_{0}$ из $(35)$, полагая

$$
b_{m-k}(\varepsilon)=\int_{\varepsilon}^{\pi-\varepsilon} q^{\prime}(\alpha) \sin (m-k) \alpha d \alpha
$$

будем иметь

$$
\begin{aligned}
A_{0}(k, \varepsilon)= & -\frac{1}{16 \pi^{3}} \sum_{l=1}^{\left[k^{\sigma}\right]} \frac{b_{l}(\varepsilon)}{l^{2} k^{1 / 2}}\left[\frac{1}{\sqrt{k+l}}-\frac{1}{\sqrt{k-l}}\right] \\
& +\frac{1}{16 \pi^{3}} \sum_{l=\left[k^{\sigma}\right]+1}^{k-N} \frac{b_{l}(\varepsilon)}{l^{2} k^{1 / 2} \sqrt{k-l}}-\frac{1}{16 \pi^{3}} \sum_{l=\left[k^{\sigma}\right]+1}^{\infty} \frac{b_{l}(\varepsilon)}{k^{1 / 2} \sqrt{k+l}} \\
= & a_{1}^{(0)}(k, \varepsilon, \sigma)+a_{2}^{(0)}(k, \varepsilon, \sigma)+a_{3}^{(0)}(k, \varepsilon, \sigma) .
\end{aligned}
$$

Изучим асимптотику $a_{1}^{(0)}(k, \varepsilon, \sigma)$. Отметим, что это слагаемое содержит главньй член асимптотики $\alpha_{k}$. Пусть $q \in C^{2}[0, \pi]$; тогда

$$
b_{l}(\varepsilon)=\frac{O(\varepsilon)}{l}+\frac{O(1)}{l} .
$$

Из формулы Тейлора для функции $f(x)=(1+x)^{-1 / 2}-(1-x)^{-1 / 2}$ при $x=l / k$ следует, что $f(l / k)=-l / k+O\left(l^{2} / k^{2}\right)$, откуда из $(36),(37)$ получим

$$
a_{1}^{(0)}(k, \varepsilon, \sigma)=\frac{1}{32 \pi^{3} k^{2}} \int_{0}^{\pi} q^{\prime}(\alpha)(\pi-\alpha) d \alpha+\frac{O\left(\varepsilon^{2}\right)}{k^{2}}+\frac{O(1)}{k^{2+\sigma}}+\frac{O(\ln k)}{k^{3}} .
$$

Мы здесь воспользовались следующими соотношениями:

$$
\left[\int_{0}^{\varepsilon}+\int_{\pi-\varepsilon}^{\pi}\right] q^{\prime}(\alpha)(\pi-\alpha) d \alpha=O\left(\varepsilon^{2}\right), \quad \sum_{l=\left[k^{\sigma}\right]+1}^{\infty} \frac{b_{l}}{k^{2} l}=\frac{O(1)}{k^{2+\sigma}}, \quad \sum_{l=1}^{\infty} \frac{\sin l \alpha}{l}=\frac{\pi-\alpha}{2}
$$

$0<\alpha<2 \pi$, и равномерной сходимостью последнего ряда на отрезке $[\varepsilon, \pi-\varepsilon]$. Далее, используя равенство (37), нетрудно убедиться, что

$$
a_{2}^{(0)}(k, \varepsilon, \sigma)=\frac{O(1)}{k^{1+2 \sigma}}, \quad a_{3}^{(0)}(k, \varepsilon, \sigma)=\frac{O(1)}{k^{1+2 \sigma}} ;
$$

следовательно, в силу $(36)$ для $A_{0}(k, \varepsilon)$ с учетом замечания 2 получим следующую асимптотику:

$$
A_{0}(k, \varepsilon)=\frac{1}{32 \pi^{3} k^{2}} \int_{0}^{\pi} q^{\prime}(\alpha)(\pi-\alpha) d \alpha+\frac{O\left(\varepsilon^{2}\right)}{k^{2}}+\frac{O(1)}{k^{1+2 \sigma}} .
$$


Для второго слагаемого в (33) имеем

$$
\begin{aligned}
A_{1}(k, \varepsilon)= & -\frac{\tau_{1} k^{1 / 2}}{32 \pi^{2}} \sum_{l=1}^{\left[k^{\sigma}\right]} \frac{b_{l}(\varepsilon)}{l^{2}}\left[\frac{\sqrt{k+l}}{2 k+l}-\frac{\sqrt{k-l}}{2 k-l}\right] \\
& +\frac{\tau_{1} k^{1 / 2}}{32 \pi^{2}} \sum_{l=\left[k^{\sigma}\right]+1}^{k-N} \frac{b_{l}(\varepsilon)}{l^{2}} \frac{\sqrt{k-l}}{2 k-l}-\frac{\tau_{1} k^{1 / 2}}{32 \pi^{2}} \sum_{l=\left[k^{\sigma}\right]+1}^{\infty} \frac{b_{l}(\varepsilon)}{l^{2}} \frac{\sqrt{k+l}}{2 k+l} \\
= & \sum_{i=1}^{3} a_{i}^{(1)}(k, \varepsilon, \sigma) .
\end{aligned}
$$

Применяя формулу Тейлора для функции

$$
f(x)=\frac{\sqrt{1+2 x}}{1+x}-\frac{\sqrt{1-2 x}}{1-x}
$$

при $x=l /(2 k)$, получаем, что

$$
f\left(\frac{l}{2 k}\right)=\frac{l^{3}}{4 k^{3}}+O\left(\frac{l^{4}}{k^{4}}\right)
$$

Поэтому, используя $(20)$ при $\varphi(\alpha)=1$ для первого слагаемого в (39), находим

$$
a_{1}^{(1)}(k, \varepsilon, \sigma)=\frac{O(\varepsilon)}{k^{3-\sigma}} .
$$

Для второго и третьего слагаемых в (39), воспользуясь оценкой (20), легко установим, что

$$
a_{2}^{(1)}(k, \varepsilon, \sigma)=\frac{O(\varepsilon)}{k^{2 \sigma}}+\frac{O(1)}{k^{3 \sigma}}+\frac{O(\varepsilon)}{k^{2}}, \quad a_{3}^{(1)}(k, \varepsilon, \sigma)=\frac{O(\varepsilon)}{k^{2 \sigma}}+\frac{O(1)}{k^{3 \sigma}} .
$$

Таким образом, с учетом замечания 2 (так как $\sigma \in(0,1))$ находим, что

$$
A_{1}(k, \varepsilon)=\frac{O(\varepsilon)}{k^{2 \sigma}}+\frac{O(1)}{k^{3 \sigma}}+\frac{O(\varepsilon)}{k^{2}} .
$$

Далее, производя совершенно аналогичные выкладки как при исследовании $A_{1}(k, \varepsilon)$, замечая при этом, что

$$
f(x)=\frac{1+x}{(1+2 x)^{3 / 2}}-\frac{1-x}{(1-2 x)^{3 / 2}}
$$

для $A_{2}(k, \varepsilon)$ и

$$
f(x)=\frac{1}{(1+x) \sqrt{1+2 x}}-\frac{1}{(1-x) \sqrt{1-2 x}}
$$

для $A_{3}(k, \varepsilon)$, с учетом замечания 2 находим

$$
A_{2}(k, \varepsilon)=O\left(\frac{1}{k^{3}}\right)+\frac{O(\varepsilon)}{k^{2+2 \sigma}}+\frac{O(1)}{k^{2+3 \sigma}}, \quad A_{3}(k, \varepsilon)=O\left(\frac{1}{k^{3}}\right)+\frac{O(\varepsilon)}{k^{2+2 \sigma}}+\frac{O(1)}{k^{2+3 \sigma}}
$$


Теперь изучим слагаемое $B_{1}(k, \varepsilon)$ из (34). Оно совпадает с $A_{3}(k, \varepsilon)($ см. $(33)$ и $(34))$, разнища лишь в том, что в знаменателе под интегралом появляется $\sin ^{2} \alpha$. Следовательно, используя оценку (20) при $n=2$, нетрудно убедиться, что

$$
B_{1}(k, \varepsilon)=\frac{O(\ln \varepsilon)}{k^{2+\sigma}} .
$$

Для того, чтобы изучить поведение $A_{4}(k, \varepsilon)$ и $B_{2}(k, \varepsilon)$ при $k \rightarrow \infty$, воспользуемся следуюшим равенством:

$$
\frac{m^{3}+k^{3}}{k^{3 / 2} m^{3 / 2}(m+k)^{2}}=-\frac{1}{k^{1 / 2} m^{3 / 2}(m+k)}+\frac{m^{1 / 2}}{m^{3 / 2}(m+k)}+\frac{k^{1 / 2}}{m^{3 / 2}(m+k)} .
$$

Тогда части $A_{4}(k, \varepsilon)$ и $B_{2}(k, \varepsilon)$, соответствующие первому слагаемому в $(43)$, совпадают с $A_{3}(k, \varepsilon)$ и $B_{1}(k, \varepsilon)$ соответственно. А для частей $A_{4}(k, \varepsilon)$ и $B_{2}(k, \varepsilon)$, соответствующих второму и третьему слагаемым, применяя общую схему исследования (при этом, используя равенство (37) и оценку (19) соответственно), в силу сказанного выше с учетом (41) и (42) получаем

$$
A_{4}(k, \varepsilon)=\frac{O(1)}{k^{3}}+\frac{O(1)}{k^{2+2 \sigma}}, \quad B_{2}(k, \varepsilon)=\frac{O(\ln \varepsilon)}{k^{5 / 2}}+\frac{O(\ln \varepsilon)}{k^{2+\sigma}} .
$$

Отметим, что $A_{4}(k, \varepsilon)$ в $(44)$ не зависит от $\varepsilon$ из замечания 2 .

Теперь с учетом замечания 2 из равенств (33) и (34) и оценок $(38),(40)-(42),(44)$ заключаем, что

$$
\begin{aligned}
\alpha_{k}^{(2,8)}(\varepsilon)= & \frac{1}{32 \pi^{3} k^{2}} \int_{0}^{\pi} q^{\prime}(\alpha)(\pi-\alpha) d \alpha \\
& +\frac{O(\varepsilon)}{k^{2}}+\frac{O(1)}{k^{1+2 \sigma}}+\frac{O(\varepsilon)}{k^{2 \sigma}}+\frac{O(1)}{k^{3 \sigma}}+\frac{O(1)}{k^{5 / 2}}+\frac{O(\varepsilon)}{k^{2+2 \sigma}}, \quad 0<\sigma<1, \\
\alpha_{k}^{(2,9)}(\varepsilon)= & \frac{O(\ln \varepsilon)}{k^{5 / 2}}+\frac{O(\ln \varepsilon)}{k^{2+\sigma}}, \quad 0<\sigma<1 .
\end{aligned}
$$

Положим $\sigma=3 / 4$. Тогда, так как

$$
\alpha_{k}^{(2)}=\sum_{i=1}^{9} \alpha_{k}^{(2, i)}(\varepsilon)
$$

то из оценок $(21)-(25),(31),(32),(45),(46)$ вытекает утверждение леммы 4.

Теперь если положим $\varepsilon=k^{-\delta}, 2 / 3<\gamma<3 / 4$, то в силу равенства (13) из лемм $2-4$ получим

$$
\alpha_{k}=\frac{1}{32 \pi^{3} k^{2}} \int_{0}^{\pi} q^{\prime}(\alpha)(\pi-\alpha) d \alpha+o\left(\frac{1}{k^{2}}\right) .
$$

Преобразуем интеграл в (47). Так как

$$
\int_{0}^{\pi} q^{\prime}(\alpha)(\pi-\alpha) d \alpha=\int_{0}^{\pi} q(\alpha) d \alpha-\pi q(0)
$$


а из формул $(5),(7)$ и определения вектора $\widetilde{\omega}$ получаем, что

TO

$$
q(0)=2 \pi \int_{\mathbb{S}^{2}} V^{2}\left(\omega_{0}\right) d \omega_{0}
$$

$$
\begin{aligned}
\int_{0}^{\pi} q(\alpha) d \alpha & =\int_{0}^{\pi} \int_{0}^{2 \pi} \int_{\mathbb{S}^{2}} \frac{V\left(\omega_{0}\right) V\left(T\left(\omega_{0}\right) \widetilde{\omega}\right) d \omega_{0} \sin \alpha d \psi d \alpha}{\sin \alpha} \\
& =\int_{\mathbb{S}^{2}} \int_{\mathbb{S}^{2}} \frac{V\left(\omega_{0}\right) V(\omega)}{\sqrt{1-\left(\omega, \omega_{0}\right)^{2}}} d \omega d \omega_{0}
\end{aligned}
$$

(в последнем равенстве мы воспользовались тем, что $\sin \alpha=\sqrt{1-\left(\omega, \omega_{0}\right)^{2}}$ заменой переменных $\omega=T\left(\omega_{0}\right) \widetilde{\omega}$ с якобианом равным 1$)$. Таким образом, из (47) и последних преобразований следует теорема 2, а из нее - доказательство теоремы 1.

В работе [7, теорема 3] при $V \in C^{2}\left(\mathbb{S}^{2}\right)$ была доказана оценка $\alpha_{k}=o\left(k^{-1}\right)$, которую при этих же услових на $V$ легко усилить, а именно, имеет место

ЛЕмма 5. При $k \gg 1 u V \in C^{2}\left(\mathbb{S}^{2}\right)$

$$
\alpha_{k}=O\left(k^{-\lambda}\right), \quad \lambda>1 \text {. }
$$

ДоКАЗАТЕЛЬСТВО. При $q \in C^{2}[0, \pi]$ (в силу формулы $(7)$ гладкости $q$ и $V$ совпадают) леммы 2 и 3 справедливы. При доказательстве леммы 4 условие, что $q \in C^{3}[0, \pi]$, т.е. оценку $(20)$, использовали лишь при уточнении асимптотики слагаемых $\alpha_{k}^{(2,4)}(\varepsilon)$ $($ см. $(24)), \alpha_{k}^{(2,6)}(\varepsilon)($ см. $(31))$ и $A_{1}(k, \varepsilon)$ (см. (40)). Пусть $q \in C^{2}[0, \pi]$. Тогда, используя оценку

$$
\int_{\varepsilon}^{\pi-\varepsilon} q^{\prime}(\alpha)[\sin \alpha \sin (m+k) \alpha+\cos (m+k) \alpha] d \alpha=\frac{O(1)}{m+k}
$$

вместо $(20)$ для $\alpha_{k}^{(2,4)}(\varepsilon)$, равенство (37) вместо $(20)$ для $\alpha_{k}^{(2,6)}(\varepsilon)$ и $A_{1}(k, \varepsilon)$, учитьвая замечание 2 , находим, что

$$
\alpha_{k}^{(2,4)}(\varepsilon)=\frac{O(1) \ln k}{k^{2}}, \quad \alpha_{k}^{(2,6)}(\varepsilon)=\frac{O\left(\varepsilon^{-1}\right)}{k^{2+\sigma}}+\frac{O\left(\varepsilon^{-1}\right)}{k^{5 / 2-\sigma}}, \quad A_{1}(k, \varepsilon)=\frac{O(1)}{k^{2 \sigma}} .
$$

Из лемм 2 и 3 и оценок для $\alpha_{k}^{(2, i)}(\varepsilon), i=1, \ldots, 9$, с учетом (48) и замечания 2 получим

$$
\alpha_{k}=O\left(\varepsilon^{3} \ln k\right)+\frac{O\left(\varepsilon^{3 / 2}\right)}{k^{3 / 2}}+\frac{O(1) \ln k}{k^{2}}+\frac{O\left(\varepsilon^{-1}\right)}{k^{2+\sigma}}+\frac{O(1)}{k^{2 \sigma}}+\frac{O\left(\varepsilon^{-2}\right)}{k^{7 / 2}},
$$

откуда, полагая $\sigma=3 / 4$ и $\varepsilon=k^{-\gamma}, 2 / 3<\gamma<3 / 4$, получим доказательство леммы 5 .

Теперь в силу этой леммы и теоремы 1 работы [7] заключаем, что справедлива

ТЕорема 3. Пусть $V$ - оператор умножения, вообще говоря, на комплексную функиию из класса $C^{2}\left(\mathbb{S}^{2}\right)$ в пространстве $L_{2}\left(\mathbb{S}^{2}\right)$. Тогда для собственных чисел оператора L справедливо тохсество

$$
\sum_{k=0}^{\infty} \sum_{i=-k}^{k}\left[\mu_{k i}-k(k+1)-c_{0}\right]=2 c_{1}
$$

постоянные $c_{0}$ и $c_{1}$ определены в (2), причем ряд в (49) сходится абсолютно.

ЗАмЕчАнИЕ 3 . Из формул (2), (49) и теоремы 2 следует, что регуляризованньй след возмущения оператора Лапласа на $\mathbb{S}^{2}$ равен удвоенному коэффициенту главного члена второй поправки теории возмущений, которьй является дисперсией от возмущения $V$. 


\section{СПИСОК ЦИТИРОВАННОЙ ЛИТЕРАТУРЫ}

[1] Guillemin B. Some spectral results for the Laplace operator with potential on the $n$-sphere // Adv. in Math. 1978. V. 27. P. 273-286.

[2] Widom H. The Laplace operator with potential on the 2-sphere // Adv. in Math. 1979. V. 31. P. 63-66.

[3] Садовничий В. А., Дубровский В. В. Классическая формула регуляризованного следа для собственных чисел оператора Лапласа-Бельтрами с потенциалом на сфере // Докл. АН CССР. 1991. Т. 319. №1. С. 61-62.

[4] Подольский В.Е. Формула регуляризованного следа оператора Лапласа-Бельтрами с нечетным потенциалом на сффере $\mathbb{S}^{2} / /$ Матем. заметки. 1994. Т. 56. № 1. С. 71-77.

[5] Фазуллин 3. Ю. Регуляризованный след оператора Лапласа-Бельтрами // Международная конференция по комплексному анализу и смежньм вопросам. Тезисы докл. Нижний Новгород, 1997. С. 80-81.

[6] Бобров А. Н. След оператора Лапласа-Бельтрами с потенциалом на поверхности Цолля // Докл. РАН. 1999. Т. 368. № 2. С. 154-156.

[7] Садовничий В.А., Фазуллин З. Ю. Формула первого регуляризованного следа для возмущения оператора Лапласа-Бельтрами // Дифференц. уравнения. 2001. Т. 37. № 3 . C. $402-409$.

[8] Садовничий В. А., Дубровский В. В., Порецков О. А. Формула первого регуляризованного следа оператора Лапласа-Бельтрами с негладким потенциалом на двумерной сфере // Докл. РАН. 2002. Т. 382. №1. С. 11-14.

[9] Садовничий В. А., Фазуллин З. Ю. Кластерная асимптотика собственных чисел возмущения оператора Лапласа на сфере $\mathbb{S}^{2}$ // Докл. РАН. 2003. Т. 391. № 4. С. 456-459.

[10] Гобсон Е. В. Теория сферических и эллипсоидальных функций. М.: ИЛ, 1952.

[11] Сегё Г. Ортогональные многочлены. М.: ГИФМЛ, 1962.

[12] Олвер Ф. Асимптотика и специальные функции. М.: Наука, 1990.

(В. А. Садовничий) Московский государственный университет

Поступило им. М. В. Ломоносова

18.11.2003

(З. Ю. Фазуллин) Башкирский государственный университет, г. Уфа

E-mail: fazullinzu@mail.ru

Исправленный вариант

08.07.2004 